
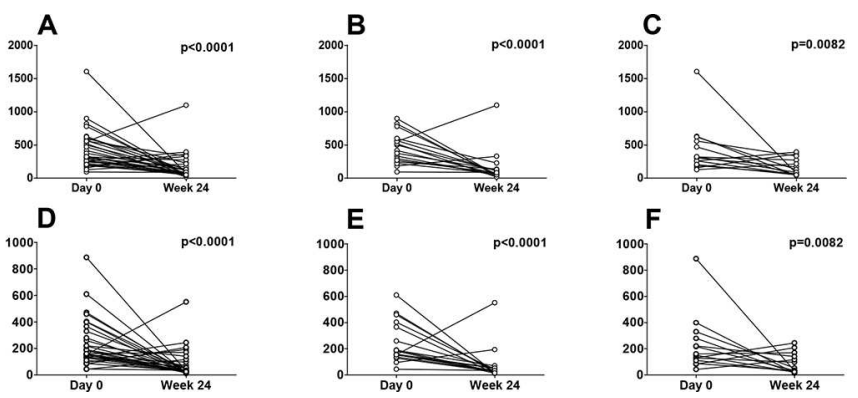

Results In the child cohort passive smoking was found to be an independent risk factor for Mtb infection (OR: 1.52, 95\% CI: $1.09-2.12)$. Higher household monthly income was an independent protective factor against Mtb infection (OR: 0.55, 95\% CI: $0.38-0.79)$. Increasing age was associated with a decreased risk of progressing from infection to disease (OR: 0.67, 95\% CI: 0.51-0.87). Children exposed to more than $1 \mathrm{~TB}$ patient were 8 times more likely to progress to disease (OR: 8.66 , 95\% CI: 1.54-48.55).

Conclusion Identification of an association between passive exposure to cigarette smoke and acquisition of Mtb infection in children adds new evidence for smoking cessation strategies to

Abstract S59 Figure 1. Concentration of small RNA in blood plasma before (Day 0) and after (week 24) treatment for individuals with active pulmonary tuberculosis and who were culture-negative at week $24(n=31)$ : A-C Small RNA concentration (6-150 nucleotides), D-F miRNA concentration (10-40 nucleotides). A and $D$ all individuals, $B$ and $E$ are HIV-1 negative $(n=17)$ and $C$ and $F$ are co-infected with HIV-1 $(n=14)$.

plasma small RNA (0-150 nucleotides) concentration was significantly higher $(\mathrm{p}<0.0001)$ in 31 individuals before therapy (median 332pg $\mu \mathrm{L}^{-1}$ plasma, range 93-1603pg $\mu \mathrm{L}^{-1}$ ) than at the end of therapy at week 24 (median $86 \mathrm{pg} \mu \mathrm{L}^{-1}$ plasma, range 16$1098 \mathrm{pg}_{\mu} \mathrm{L}^{-1}$ ). Expression analysis of small RNA genes revealed that, in 5 tuberculosis-infected HIV-1 negative individuals, 36 of 90 genes $(>2$-fold, $\mathrm{p}<0.05)$ were upregulated before compared to post-therapy completion. Hsa-miR-19b, 29a, 17-3p, 133a, small RNA concentration and SNORD61 were further tested and this analysis revealed that in $84 \%$ of individuals $(\mathrm{n}=31)$ at least one of these biomarkers was upregulated $>2$ fold in active tuberculosis. Co-infection with HIV-1 was not found to change the expression of these six tested biomarkers.

\section{S60 RISK FACTORS ASSOCIATED WITH MYCOBACTERIUM TUBERCULOSIS (MTB) INFECTION AND PROGRESSION TO ACTIVE TB DISEASE IN CHILD CONTACTS}

N Karnani, S Sridhar, D Connell, A Lalvani; Imperial College London, London, United Kingdom

\subsection{6/thoraxjnl-2013-204457.67}

Background Mathematical modelling has shown the most effective strategy to eliminate tuberculosis (TB) worldwide is to address the large burden of latent TB infection (LTBI). Identification of the risk factors which predispose individuals to acquire $\mathrm{Mtb}$ infection and those determining risk of progressing from infection to active disease will enable risk stratification for targeted TB interventions.

Objective To identify host, socioeconomic and environmental risk factors for acquiring Mtb infection following exposure to $\mathrm{TB}$ and risk factors for progression from infection to active TB disease.

Methods Risk factors associated with infection and progression were investigated in a primary analysis of a well-defined cohort of 965 Turkish household child contacts exposed to smear positive pulmonary TB patients. Risk factors for infection were assessed in study subjects with and without Mtb infection. Mtb infection was defined by interferon gamma-release assay (IGRA) results at two time points-baseline and 6 months-thus creating robust criteria to avoid misclassification of IGRA converters and IGRA reverters. Adjusted odd ratios were estimated using stepwise logistic regression including variables with $p<0.2$ on univariate regression. be incorporated into TB prevention programmes. To aid TB elimination we therefore advocate an enforcement of stricter tobacco control policies, particularly in regions with a high burden of TB.

\section{S61 SERIAL IGRA TESTING TO IDENTIFY RECENTLY ACQUIRED LTBI IN CONTACTS OF SMEAR POSITIVE PULMONARY TUBERCULOSIS}

R Verma, H Patel, H Thuraisingam, G Woltmann, P Haldar; University Hospitals of Leicester, Leicester, UK

\subsection{6/thoraxjnl-2013-204457.68}

Introduction We have previously reported prospective data of TB risk in close contacts of tuberculosis with single step interferon gamma release assay (IGRAs) screening 8-12 weeks after index notification and shown a lower positive predictive value of the test in contacts with a higher risk of prior exposure and remote latent tuberculosis infection (LTBI).

We hypothesised that a dynamic change in IGRA response using a two step approach may better identify recent LTBI.

Method Two-step IGRA screening with QuantiFERON TB Gold-In Tube (QFT) was implemented in adult ( $>16$ years) contacts of smear positive pulmonary TB at Leicester. QFT testing was performed as soon as possible after index notification (T1) and if negative repeated after 8-12 weeks (T2). Quantitative QFT values were recorded in all contacts and in those having two tests, the change calculated. Data was mapped to the contacts' risk of remote infection (low, moderate, high) and estimated duration of exposure to the index case based on the reported date of symptoms onset.

Results 397 contacts of 46 smear positive TB cases were identified. 116 children were excluded. 49 adults did not attend for testing (17.4\%). 100 contacts (43\%) were QFT positive at T1. In regression analysis duration of index symptoms was independently associated with this outcome $(p=0.001)$. ROC curve analysis in subgroups stratified by risk of remote LTBI demonstrated the strongest association to be in the lowest risk group (AUC $=0.93, \mathrm{p}<0.0001$ ), with no significant association in the high risk group (AUC $=0.57, \mathrm{p}=0.404$ ). 132 contacts were QFT neg at T1 and 109 returned for repeat testing. For the persistently QFT neg group the change in QFT was \pm 1 $\log 10$ fold between visits. 9 (8.2\%) contacts seroconverted. Two subgroups were identified based on the magnitude of change. In 4 contacts the change was within $1 \log$ fold. In the remainder the change was $\geq 2 \log$ fold and all in this subgroup seroconverted with 150 days of symptoms onset in the index.

Conclusions Serial IGRA with $\geq 2 \log 10$ fold change may indicate recent infection. This may be detectable in contacts of index cases with an early diagnosis. 